\title{
PI control system design for Electromagnetic Molding Machine based on Linear Programing
}

\author{
Takayuki Ishizaki, Kenji Kashima, Jun-ichi Imura*, Atushi Katoh and Hiroshi Morita**
}

\begin{abstract}
In this paper, we design a PI controller for an electromagnet placed inside a molding machine. The eddy currents inside the electromagnet are a spatially-distributed phenomenon that is difficult to capture by using finite-dimensional systems. First, we show that the theoretical model, expressed by partial differential equations, cannot perfectly capture the properties of the actual machine. Second, we design the PI control system via a loop-shaping by directly using the data of the frequency response obtained from the experiment of the machine. The design problem is reduced to a linear programing problem.
\end{abstract}

\section{INTRODUCTION}

In molding fabrication, machines which generate mold clamping forces are called molding machines. In the past, molding machines driven by oil pressure were dominant. Recently, electric molding machines are widely used to improve controllability and cycling time of jobs. Furthermore, electromagnetic molding machines, driven by electromagnets, are currently proposed [1]. In the machines, a suction force generated by the electromagnets is directly transmitted to a mold in contrast to general electric molding machines, in which a thrust force generated by a rotary motor is transmitted through amplification. As a consequence, the electromagnetic molding machines are capable of more precise molding fabrication.

However, it is difficult to realize desired force responses using heuristic tunings of controllers. The reason is that eddy currents are spatially distributed in an iron core of the electromagnet. This means that the system should be treated as a distributed parameter system [5], [10], [11], [13]. Therefore, the system is difficult to model in finite dimensions while keeping suitable properties for control system design.

In this paper, we first overview a model expressed by partial differential equations (PDEs) to analyze the property of this system. However, from the analysis it is made clear that the theoretical model cannot perfectly capture the properties of the actual machine due to a certain element of a time delay included in the machine. Therefore, we design a PI control system via loop-shaping by directly using the data of the frequency response obtained from the experiment of the machine. The design problem can be reduced to a linear programing problem because the structure of the controller is restricted to PI controllers (or PI controllers and filters) for industrial applications.

*Graduate School of Information Science and Engineering, Tokyo Institute of Technology; 2-12-1, Meguro ward, Tokyo \{ishizaki,kashima, imura\}@cyb.mei.titech.ac.jp

**Sumitomo Heavy Industries, Ltd; 19, Natushima, Yokosuka city, Kanagawa $\{$ Ats_Katoh, hrh_morita\}eshi.co.jp
This paper is organized as follows. In section 2, we explain the features of an electromagnetic molding machine and the difficulty of the control system design using heuristic tuning. Furthermore, we introduce an expression of the system as PDEs and analytically characterize the structure of the system in the frequency domain. Then, this model is compared to the experimental data. In section 3, we design a PI control system via loop-shaping by directly using the experimental data of the machine. In addition, we show the time responses of the machine when using the controllers designed optimally and discuss a trade-off relation between the performance and robustness of the controlled system. Finally, we examine the improvement of the performance of the controlled system when adding a first order filter to PI controller.

\section{EleCtromagnetic MOLDING MACHINE}

\section{A. Summary of the molding machine}

Fig. 1 shows a prototypical system of molding machines. This system consists of electromagnets, generating molding forces, a center rod, transmitting forces generated at the electromagnets, a clamping mechanism, performing the molding and a frame, holding the whole system. The mold clamping mechanism consists of the mold and a load cell, which measures molding forces. Fig. 2 shows the electromagnets placed inside the system. The electromagnets consist of an electromagnet core, a suction plate and a coil. They generate magnetic flux, shown in Fig. 2, by the current flowing in the coil. Sucking forces are generated by the magnetic flux at a gap between the electromagnet core and the suction plate. Then, they are transmitted to the mold located inside the mold clamping mechanism, which is connected to the suction plate throughout the center rod.

\section{B. Tuning of PI control system}

To control the electromagnetic molding machine, the responses of the molding force need to settle in a short time without overshoot. Fig. 3 shows a block diagram of the PI control system. In this figure, the electromagnetic molding machine, shown in Fig. 1, is represented by the electro magnet block. The filter will be added to PI controller in order to examine the improvement of the performance (See section III-C for detail). Fig. 4 shows experimental results of the step response of the molding force ${ }^{1}$ when we vary a proportional gain of the control system. Generally, the convergent rate to a target value is larger as the proportional

\footnotetext{
${ }^{1}$ In this paper, the sampling times of the experiment were set at $1.5 \mathrm{kHz}$
} 


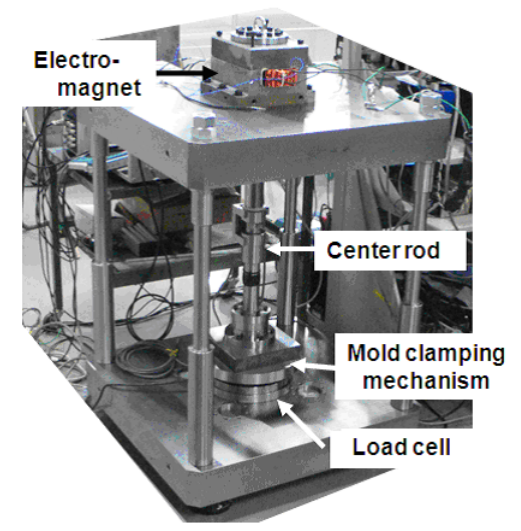

Fig. 1. Prototype system

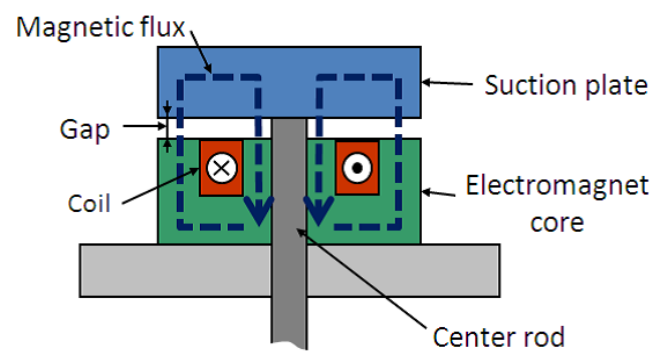

Fig. 2. Electromagnet system

gain of PI control systems is enlarged and when the gain reaches some threshold, overshoots of the response will arise. However, in the case of the electromagnetic molding machine, we can see from Fig. 4 that the convergent rate does not increase even if the gain is enlarged.

From these experiments, we can see that the behavior of this machine contradicts the intuition of general systems. Therefore, it can be anticipated that the performance of the machine will be hard to improve with the traditional tuning techniques of controllers [2]. To design the control system systematically, we first derive the mathematical expression of the system.

\section{Derivation of the expression as partial differential equa- tions}

The physical system is supposed to be axisymmetric as shown in Fig. 5. Therefore, we aim to derive relations of physical quantity for a radial direction from basic laws of physics [3]. To this end, we consider a micro region $\mathcal{D}$ along a path of the eddy current located at the radius $r$. Here, $\Gamma$ denotes a radius of the iron core.

Using magnetomotive force $E_{M}(t, r)$, magnetic resistance $R_{M}(r)$ and circumferential length $l(r)=2 \pi r$, the magnetic flux $\phi(t, r)$ generated in $\mathcal{D}$ is given by

$$
\phi(t, r)=\frac{E_{M}(t, r)}{R_{M}(r)}
$$

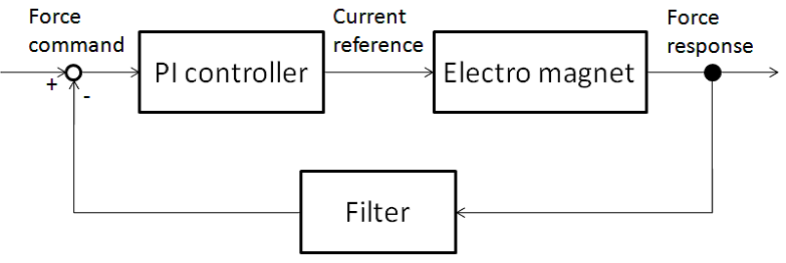

Fig. 3. PI control system

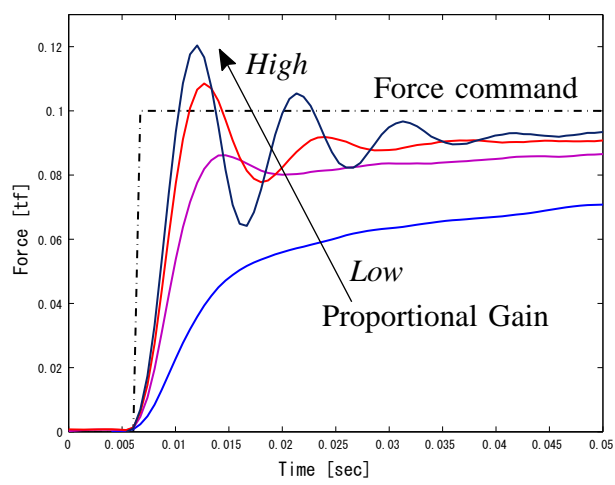

Fig. 4. Step response of electromagnet system

and

$$
R_{M}(r)=\frac{\sigma}{l(r)}, \sigma:=\left(\frac{L}{\mu_{0} \mu_{s}}+\frac{d}{\mu_{0}}\right),
$$

where $L, \mu_{0}$ and $\mu_{s}$ denote the length of the flux path, the magnetic permeability in a vacuum and the relative magnetic permeability, respectively.

Next, using induced electromotive force $E_{E}(t, r)$ and electrical resistance $R_{E}(r)$, the eddy current $i^{e}(t, r)$ that arises in $\mathcal{D}$ is given by

$$
i^{e}(t, r)=\frac{E_{E}(t, r)}{R_{E}(r)} .
$$

Here, the electrical resistance is proportional to the circumferential length and is inversely proportional to the width of the path and therefore is given by

$$
R_{E}(r)=\rho \frac{l(r)}{d r},
$$

where $\rho$ is a constant determined by the material. From the definition, the density of the magnetic flux $B(t, r)$ has a relation of

$$
B(t, r)=\frac{\phi(t, r)}{l(r) d r} .
$$

From Faraday's law of electromagnetic induction, the induced electromotive force $E_{E}(t, r)$ is given by

$$
E_{E}(t, r)=-\int_{0}^{r} l(\zeta) \frac{\partial B(t, \zeta)}{\partial t} d \zeta
$$

i.e., the summation of the time derivatives of the magnetic flux. The magnetomotive force $E_{M}(t, r)$ in $\mathcal{D}$ is given as the summation of the eddy currents $i^{e}(t, r)$ that arises outside 
$\mathcal{D}$ and the current in the coil $i_{c}(t)$. Therefore, it is expressed as

$$
E_{M}(t, r)=\int_{r}^{\Gamma} \frac{E_{E}(t, \zeta)}{\rho l(\zeta)} d \zeta+N_{c} i_{c}(t),
$$

where $N_{c}$ denotes the number of coil turns.

Hence, organizing these relations for $B(t, r)$ yields

$$
B(t, r)=-\frac{1}{\sigma \rho} \int_{r}^{\Gamma} \frac{1}{\zeta^{\prime}} \int_{0}^{\zeta^{\prime}} \zeta \frac{\partial B(t, \zeta)}{\partial t} d \zeta d \zeta^{\prime}+\frac{N_{c}}{\sigma} i_{c}(t) .
$$

Finally, the suction force $F$ generated in the iron core is given by

$$
F(t)=\frac{1}{2 \mu_{0}} \int_{0}^{\Gamma} l(r) B(t, r)^{2} d r .
$$

Here, we define, respectively, the state variable, the input and the output by $\chi(t, r):=B(t, r), v(t):=i_{c}(t)$ and $\Upsilon(t):=F(t)$ and then linearize (9) around an operating point $(\bar{v}, \bar{\chi}, \bar{\Upsilon})$. Note that the steady-state value $\bar{\chi}$ is constant and independent of the spatial variable $r$. Then, we define the errors from the operating point by $(v, \chi, \Upsilon)$ and replace the spatial variable $r / \Gamma$ by $r$. Furthermore, by differentiation of (8) with respect to $r$ and coordinate transformation as $r=\sqrt{\xi}$ for convenience, we obtain

$$
\left\{\begin{array}{l}
\frac{\partial \hat{\chi}(t, \xi)}{\partial t}=2 \alpha \frac{\partial}{\partial \xi}\left(\xi \frac{\partial \hat{\chi}(t, \xi)}{\partial \xi}\right) \text { at } \xi \in(0,1) \\
\hat{\chi}(t, \xi)=\frac{\beta}{\alpha} v(t) \text { at } \xi=1 \\
\xi \frac{\partial \hat{\chi}(t, \xi)}{\partial \xi}=0 \text { at } \xi=0 \\
\Upsilon(t)=\int_{0}^{1} \hat{\chi}(t, \xi) d \xi
\end{array}\right.
$$

where $\hat{\chi}(t, \xi):=\chi(t, \sqrt{\xi})$, and $\alpha:=2 \sigma \rho / \Gamma^{2}$ and $\beta:=$ $2 \pi \rho N_{c}^{2} \bar{v} /\left(\sigma \mu_{0}\right) \in \mathbb{R}$ are positive constants. Therefore, the dynamics of the physical system are essentially identical to that of heat diffusion systems with a diffusivity which is proportional to the spatial variable [10], [11], [12].

The input-output transfer function of this system [6], [7], [8], [13] is given by

$$
G(s):=\frac{\beta}{\alpha} \frac{J_{1}\left(2 \sqrt{-\frac{s}{2 \alpha}}\right)}{\sqrt{-\frac{s}{2 \alpha}} J_{0}\left(2 \sqrt{-\frac{s}{2 \alpha}}\right)},
$$

where

$$
J_{k}(z)=\sum_{m=0}^{\infty} \frac{(-1)^{m}}{m !(m+k) !}\left(\frac{z}{2}\right)^{2 m+k}
$$

are Bessel functions [9].

\section{Comparison with the experimental results on the actual machine}

In this section, in order to verify the validity of the model expressed by PDEs, we compare the frequency response of the model (11) with that of the experimental result of the machine [4]. In addition, we compare it with a first order system for the examination.

Fig. 6 shows the Bode diagram of the experimental result, the theoretical model and the first order system. From this

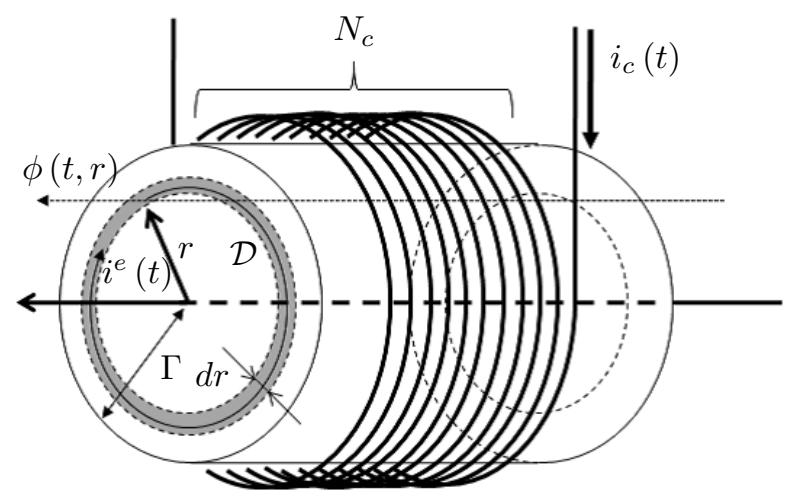

Fig. 5. Model of electromagnet

figure, we can see that the slope of the gain characteristic of the theoretical model and the experimental result is $-10[\mathrm{~dB} / \mathrm{dec}]$ at high-frequency. Generally, the slope of the gain characteristic of systems with relative degree $k$ is $-20 k[\mathrm{~dB} / \mathrm{dec}]$ at high-frequency. From this fact, we can anticipate that the property of the actual machine is hard to even approximately express in terms of a general lumped parameter system consisting of rational functions.

On the other hand, we can also see from Fig. 6 that the phase characteristics of these are different at high-frequency. This difference of the phase might be caused from certain elements of the time delay included in the machine. From this feature, we can anticipate that if we use the theoretical model for the control system design, the effect of this time delay negatively affects the behavior of the controlled system. While, we can expect that the delay can be appropriately modeled as a time-delay system. However, it is quite difficult to design the actual controller for such models, composed of PDEs and, in addition, time-delay [8].

From these examinations, we can see that the theoretical model expressed by PDEs cannot perfectly capture the property of the actual machine. Therefore, design methods in which the data of the actual machine is directly used are suitable for the control system design of this system.

\section{PI CONTROL SYSTEM DESIGN}

\section{A. PI control system design via Linear Programing}

In this section, we explain a design technique for the PI control system, shown in Fig. 3, via a Linear Programing [14].

We define the PI controller by

$$
K(s):=k_{\mathrm{P}}+\frac{k_{\mathrm{I}}}{s}
$$

and set aside the filter in Fig. 3. Furthermore, we denote the machine data of frequency response by $g_{n} \in \mathbb{C}$ and $\omega_{n} \in \mathbb{R}_{+}$, i.e., $g_{n}=G_{M}\left(j \omega_{n}\right)$ for the input-output transfer function of the machine $G_{M}(s)$, which is not explicitly expressed in this paper. Here, from the experiment of the machine, the data $g_{n}, \omega_{n}$ are obtained as Table I, in which $\omega_{1}, \cdots, \omega_{20}$ sequentially denote the values of $\omega_{n}$ from the small one and also $g_{1}, \cdots, g_{20}$ denote the values of $g_{n}$ 

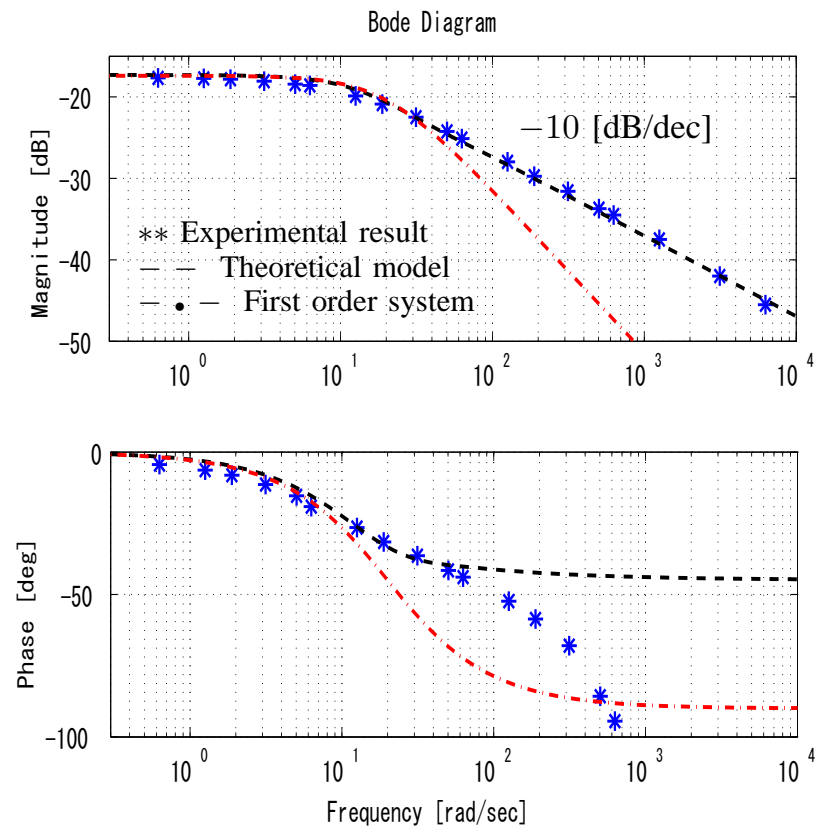

Fig. 6. Bode diagram of the machine

according to $\omega_{n}$. In the rest of this paper, we design the control system based on frequency shaping of the open-loop transfer function. Now, let us consider the following problem:

Problem: Suppose that data $g_{n} \in \mathbb{C}$ and $\omega_{n} \in \mathbb{R}_{+}$for $n=1,2, \cdots, N,\left(a_{i}, b_{i}, c_{i}\right) \in \mathbb{R}^{3}$ for $i=1,2, \cdots, I-1$ and $\left(a_{I}, b_{I}\right) \in \mathbb{R}^{2}$ are given. Furthermore suppose that the set $N_{i} \subseteq\{n=1, \cdots, N\}$ for $i=1,2, \cdots, I$ are given. Find $k_{\mathrm{P}}$ and $k_{\mathrm{I}}$ for which $L_{I}\left(j \omega_{n_{I}}\right):=g_{n_{I}} K\left(j \omega_{n_{I}}\right)$ maximize $\kappa$ such that

$$
a_{I} \operatorname{Re}\left[L_{I}\left(j \omega_{n_{I}}\right)\right]+b_{I} \operatorname{Im}\left[L_{I}\left(j \omega_{n_{I}}\right)\right]<\kappa,
$$

subject to all $L_{i}\left(j \omega_{n_{i}}\right):=g_{n_{i}} K\left(j \omega_{n_{i}}\right)$ for $i=1,2, \cdots, I-1$ satisfy

$$
a_{i} \operatorname{Re}\left[L_{i}\left(j \omega_{n_{i}}\right)\right]+b_{i} \operatorname{Im}\left[L_{i}\left(j \omega_{n_{i}}\right)\right]<c_{i}
$$

for $n_{i} \in N_{i}$.

This frequency shaping problem can be reduced to a linear programing problem, which can be solved efficiently by numerical computation, because the structure of the controller is restricted to PI controller. Therefore, we can shape the frequency property of the open-loop transfer function by using the machine data obtained from the experiment. The physical interpretation of each sets and the parameters in this problem and relation to the actual design problem are explained throughout discussion in the next sections.

\section{B. Loop shaping by using the frequency response data}

In this section, we design the PI control system and also show the time responses of the designed system. Here, we maximize $\kappa$ with several values of $\gamma$ under the constraints that $k_{\mathrm{P}}$ and $k_{\mathrm{I}}$ satisfy

$$
\begin{aligned}
& N_{1}=\{1, \cdots, 12\}, \quad\left(a_{1}, b_{1}, c_{1}\right)=(-1,1, \gamma) \\
& N_{2}=\{1, \cdots, 20\}, \quad\left(a_{2}, b_{2}, c_{2}\right)=(0,1,-\kappa) .
\end{aligned}
$$

In this optimization problem, we maximize $\kappa$ under the constraints of the half plane for the loop transfer function $L_{i}\left(j \omega_{n_{i}}\right)$ at the frequency points $\omega_{n_{i}}, n_{i} \in N_{i}$. The restriction boundaries are straight lines, which are defined by the parameters $\left(a_{i}, b_{i}, c_{i}\right)$ for $i=1,2$. To put it concretely, the first restrictions guarantee some stability margin by giving some $\gamma$ and the second restriction maximizes the gains in the low- and middle-frequency range by maximizing $\kappa$. As a result, we obtained $k_{\mathrm{P}}=9.90, k_{\mathrm{I}}=8.43 \times 10^{3}$ and $\kappa=1.95$ when $\gamma=0.1$ and $k_{\mathrm{P}}=19.8, k_{\mathrm{I}}=1.69 \times 10^{4}$ and $\kappa=3.90$ when $\gamma=0.2$.

Fig. 7 and Fig. 8 show Nyquist plots of $L_{i}\left(j \omega_{n_{i}}\right)$, which are enlarged in Fig. 9 and Fig. 10 around the origin, and that of the theoretical model (11) when using the controller obtained here. In these figures, the solid line and the broken line denote the Nyquist plot of $L_{i}\left(j \omega_{n_{i}}\right)$ and that of the theoretical model, i.e., $G(j \omega) K(j \omega)$, respectively. In addition : and $*$ denote the ranges $\omega_{n_{1}}$ for all $n_{1} \in N_{1}$ and $\omega_{n_{2}}$ for all $n_{2} \in N_{2}$, respectively. From these figures, we can see that the gains are maximized in the low- and middle-frequency range while all $L_{i}\left(j \omega_{n_{i}}\right)$ satisfy the restrictions and also that the obtained $\kappa$ becomes smaller when we give larger $\gamma$. Furthermore, the phase of $L_{i}\left(j \omega_{n_{i}}\right)$ delays more than that of the theoretical model $G(j \omega) K(j \omega)$. Therefore, we can anticipate that if we use the theoretical model for the control system design, we cannot guarantee the stability margin of the controlled system appropriately.

Furthermore, we can obtain a trade-off relation between $\kappa$ and $\gamma$, i.e., guarantee of the gain in the low- and middlefrequency range and the robustness of the closed system, as shown in Fig. 11. That is to say, we cannot improve the convergence rate without losing some of the stability margin. Consequently, we can theoretically show the performance limitation of the PI controller for this system, which is hard to determine by heuristic tuning of the controller.

In addition, Fig. 12 shows the experimental results of the time response for the controllers obtained from solving the following problem:

$$
\begin{aligned}
& N_{1}=\{1, \cdots, 16\}, \quad\left(a_{1}, b_{1}, c_{1}\right)=(-4.6,1, \gamma) \\
& N_{2}=\{1, \cdots, 20\}, \quad\left(a_{2}, b_{2}, c_{2}\right)=(0,1,-\kappa) .
\end{aligned}
$$

As a result, we obtained $k_{\mathrm{P}}=3.28, k_{\mathrm{I}}=33.5$ and $\kappa=$ 0.06 when $\gamma=0.12$ and $k_{\mathrm{P}}=6.56, k_{\mathrm{I}}=66.2$ and $\kappa=$ 0.12 when $\gamma=0.24$. We can see from this figure that both experimental results converge to the target value and also that the convergence rate improves as the value of $\gamma$ is larger.

\section{Examination for the improvement of the performance}

In section III-B, we showed the performance limitation when using the PI controller only. Besides, in this section we examine the improvement of the performance when adding a first order filter to PI controller. 
TABLE I

DATA SET OF FREQUENCY RESPONSE OF THE MACHINE

\begin{tabular}{|c||c|c|c|c|c|}
\hline$\omega_{n}[\mathrm{rad} / \mathrm{sec}]$ & 0.6283 & 1.257 & 1.885 & 3.142 & 5.027 \\
\hline$g_{n}$ & $0.1306+0.0100 j$ & $0.1291+0.0144 j$ & $0.1272+0.0182 j$ & $0.1226+0.0247 j$ & $0.1156+0.0317 j$ \\
\hline \hline & 6.283 & 12.57 & 18.85 & 31.42 & 50.27 \\
\hline & $0.1111+0.0386 j$ & $0.0907+0.0452 j$ & $0.0770+0.0473 j$ & $0.0604+0.0445 j$ & $0.0460+0.0408 j$ \\
\hline \hline & 62.83 & 125.7 & 188.5 & 314.2 & 502.7 \\
\hline & $0.0400+0.0385 j$ & $0.0244+0.0317 j$ & $0.0170+0.0278 j$ & $0.0099+0.0244 j$ & $0.0015+0.0206 j$ \\
\hline & 628.3 & 1257 & 3142 & 6283 & 12570 \\
\hline & $-0.0015+0.0188 j$ & $-0.0076+0.0109 j$ & $-0.0079+0.0000 j$ & $-0.0018-0.0050 j$ & $0.0026-0.0030 j$ \\
\hline
\end{tabular}

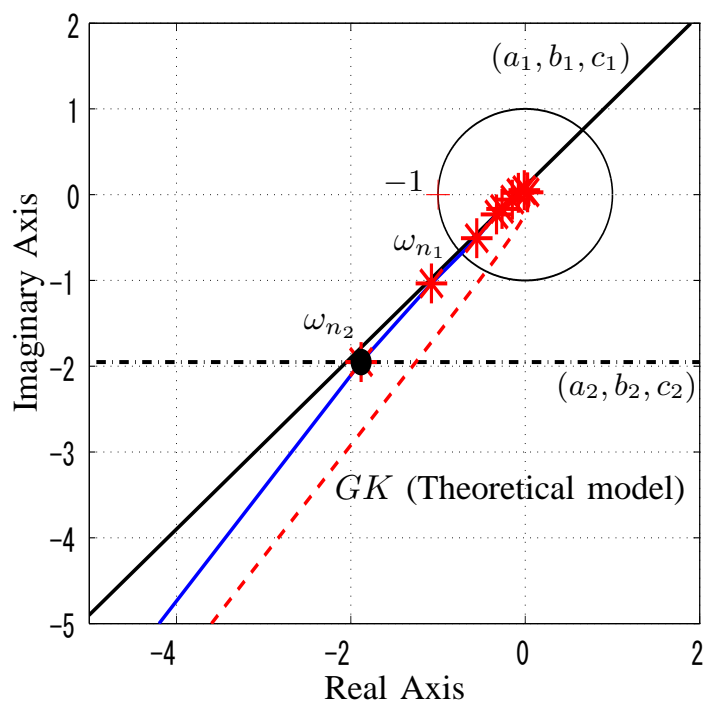

Fig. 7. Nyquist plot $(\gamma=0.1)$

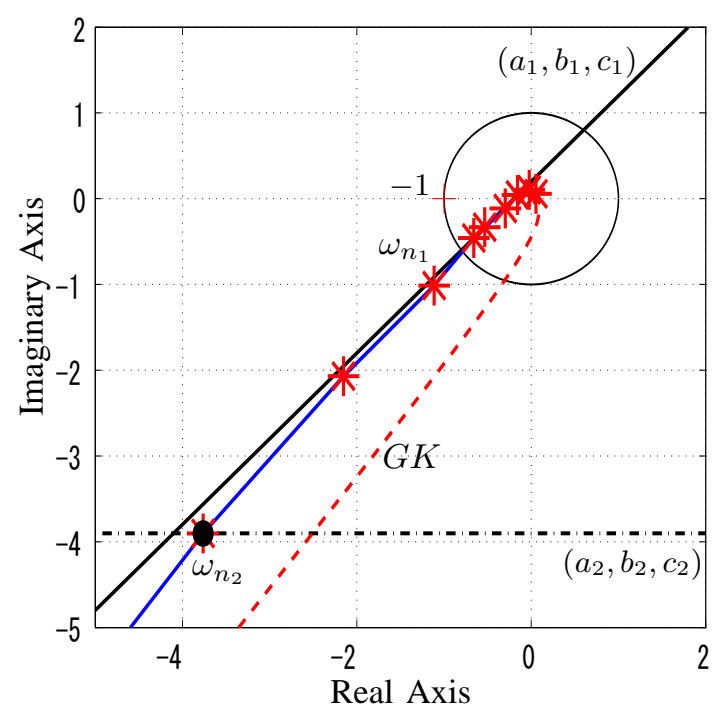

Fig. 8. Nyquist plot $(\gamma=0.2)$
In the rest of this section, we solve the same frequency shaping problems as those in section III-A for the controller of

$$
K(s):=k_{\mathrm{P}}+\frac{k_{\mathrm{I}}}{s}+\frac{k_{\mathrm{F}}}{s T+1},
$$

in which the filter is added to (13) and $T$ denotes the time constant of the filter.

Here, when $T=30$ and $\gamma=0.2$, we maximize $\kappa$ under the constraints that $k_{\mathrm{P}}, k_{\mathrm{I}}$ and $k_{\mathrm{F}}$ satisfy

$$
\begin{aligned}
& N_{1}=\{1, \cdots, 12\}, \quad\left(a_{1}, b_{1}, c_{1}\right)=(-1,1,0.2) \\
& N_{2}=\{1, \cdots, 20\}, \quad\left(a_{2}, b_{2}, c_{2}\right)=(0,1,-\kappa) .
\end{aligned}
$$

As a result, we obtained $k_{\mathrm{P}}=19.3, k_{\mathrm{I}}=-3.32 \times 10^{7}, k_{\mathrm{F}}=$ $9.98 \times 10^{8}$ and $\kappa=6.31$. Fig. 13, in which the denotations are same as those in Fig. 7 or Fig. 8, shows Nyquist plots of $L_{i}\left(j \omega_{n_{i}}\right)$ and the theoretical model $G(j \omega) K(j \omega)$ when using the controller obtained here. From this figure, we can see that the gain in the low- and middle-frequency range is bigger than that in Fig. 8 while keeping the same stability margin, i.e., $\gamma=0.2$. That is to say, the performance of the controlled system improves by increasing the order of
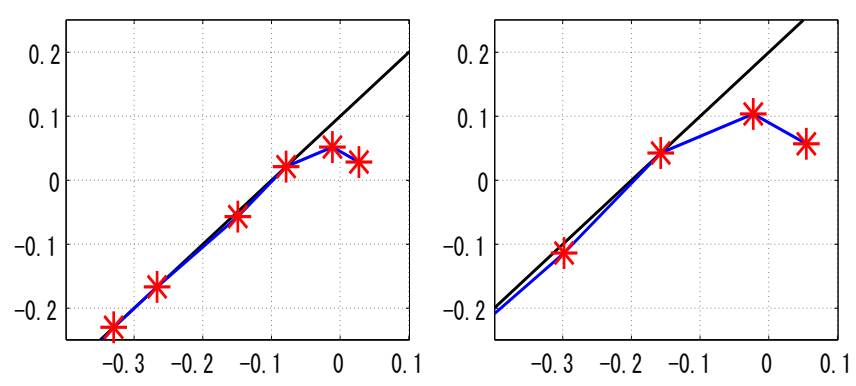

Fig. 9. Enlargement of Fig. 7

Fig. 10. Enlargement of Fig. 8

the controller. Moreover, the Nyquist plot of the theoretical model is distant from that of $L_{i}\left(j \omega_{n_{i}}\right)$.

Furthermore, Fig. 14 shows the obtained value of $\kappa$ when varying the time constant $T$. This figure indicates that the performance will improve significantly by choosing the time constant between 20 and $30(\max \kappa \simeq 9)$. However, since the obtained gains are too large to implement, we need to practically design controllers taking this point into account. 


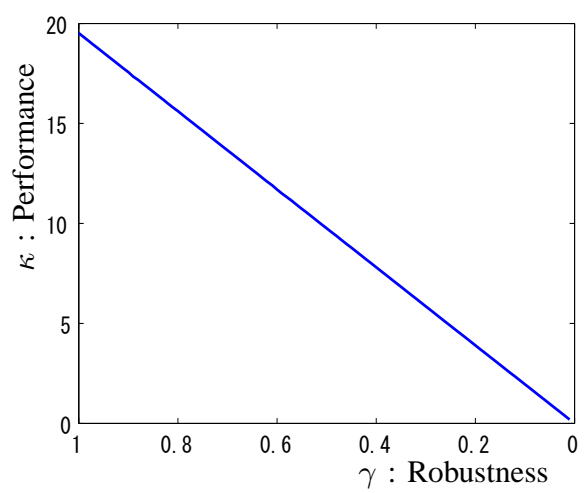

Fig. 11. Trade-off relation between $\kappa$ and $\gamma$

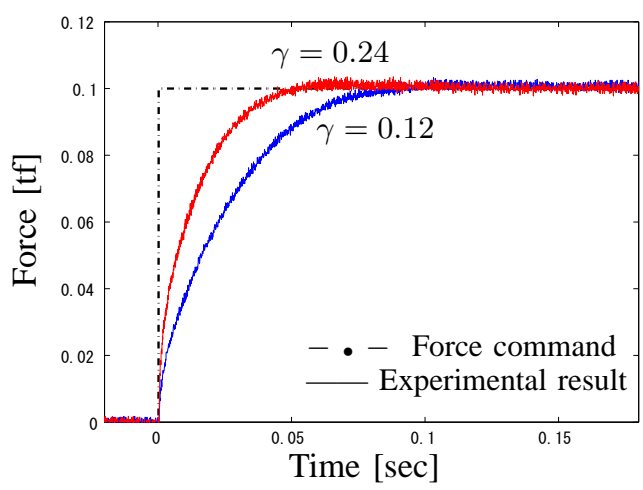

Fig. 12. Experimental result of time response

\section{CONCLUSION}

Spatially-distributed phenomena are important to industrial applications, while the mathematical treatment of them are complex. In this paper, we showed that the theoretical model expressed by PDEs was not necessarily suitable for the control system design because the model could not perfectly capture the property of the actual machine. Therefore, in this paper we designed a PI control system by directly using the experimental result via the frequency shaping. The design problem was reduced to a linear programing problem. Consequently, we theoretically showed the performance limitation, which is hard to determine by heuristic tuning of the controller.

\section{REFERENCES}

[1] H. Morita, A. Kato, T. Yamamoto and T. Shibata: WO publication, WO2009/028488

[2] Y. Nozaka: Industrial Control System and Control Apparatus, CORONA PUBLISHING CO., LTD (1995)

[3] D. K. Cheng: Field and Wave Electromagnetics Second Edition, ADDISON-WESLEY PUBLISHING COMPANY (1992)

[4] L. Ljung: SYSTEM IDENTIFICATION: Theory for the User, PRENTICE HALL PTR (1987)

[5] R. Curtain and H. Zwart: An Introduction to Infinite-Dimensional Control Theory, Springer (1995)

[6] R. Curtain and K. Morris: "Transfer functions of distributed parameter systems: A tutorial" Automatica 45 1101-1116 (2009)

[7] R. Padhi and S. F. Ali: "An account of chronological developments in control of distributed parameter systems" Annual Reviews in Control 33, 59.68 (2009)

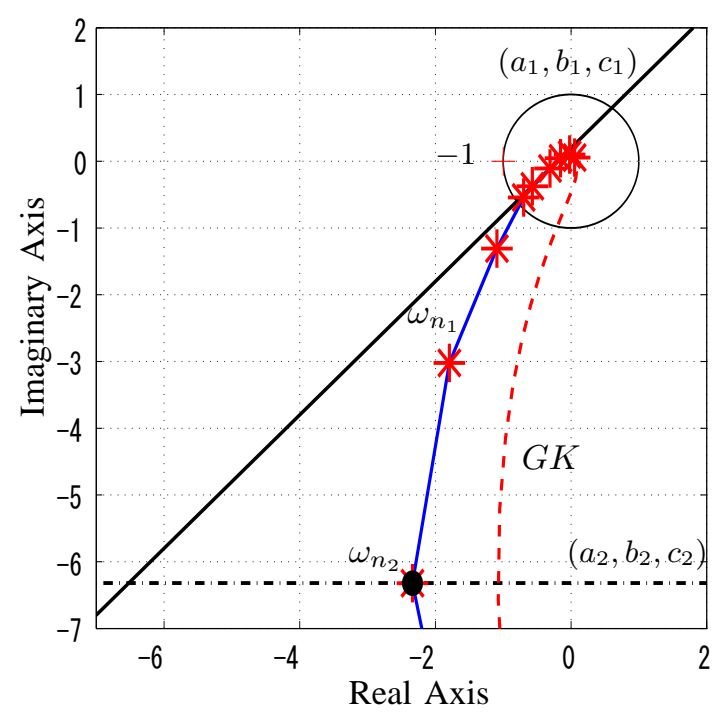

Fig. 13. Nyquist plots of the designed system

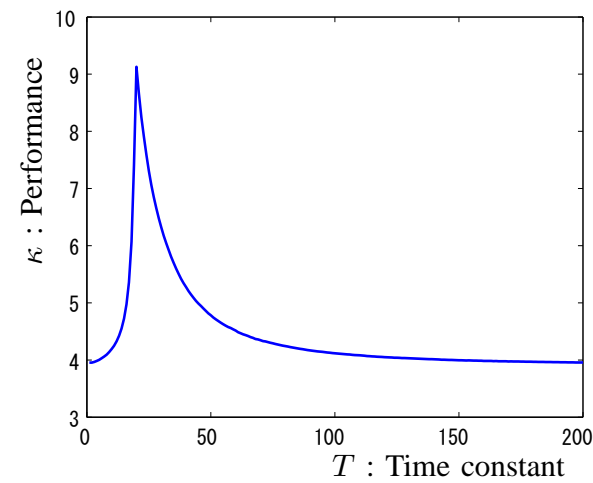

Fig. 14. Change of $\kappa$ with $T$

[8] N. Abe and A. Kojima: Control in Time-delay and Distributed Parameter Systems, CORONA PUBLISHING CO., LTD. (2007)

[9] N. W. McLachlan: Bessel Functions for Engineers Second Edition, OXFORD UNIVERSITY PRESS (1955)

[10] J. Crank: The Mathematics of Diffusion, OXFORD UNIVERSITY PRESS (1973)

[11] W. M. Deen: Analysis of Transport Phenomena, OXFORD UNIVERSITY PRESS (1998)

[12] H. S. Carslaw and J. C. Jaeger: Conduction of Heat in Solids, OXFORD SCIENCE PUBLICATIONS (1986)

[13] M. Krstic and A. Smyshlyaev: Boundary Control of PDEs - A Course on Backstepping Designs -, Society for Industrial and Applied Mathematics Philadelphia (2007)

[14] M. Kunze, A. Karimi and R. Longchamp: "Frequency domain controller design by linear programming guaranteeing quadratic stability" In Proceedings of the 47th IEEE Conference on Decision and Control, pages 345-350, Cancun, Mexico, December (2008). 